\title{
PRÁctica de BALONMANO ADAPTAdO EN UN GRUPO MIXTO EN SILLA DE RUEDAS Desde la mirada del modelo de bienestar psicológico de Ryff
}

\author{
Yisel Katherine Aristizabal Gómez, María Elena díaz Rico \\ Universidad de San Buenaventura Cali, Colombia
}

\section{PALABRAS CLAVE}

Bienestar psicológico

Discapacidad

Modelo de diversidad

Práctica deportiva

\section{RESUMEN}

Esta investigación se desarrolló con el propósito de analizar la contribución de la práctica de balonmano adaptado a nivel del bienestar psicológico (BP) posterior al proceso de entrenamiento. Se analizó el BP desde las seis categorías planteadas por Ryff (1989) Autoaceptación, Relaciones positivas con otros, Dominio del entorno, Autonomía, Propósito de vida y Crecimiento personal, estas categorías relacionadas a la experiencia deportiva por primera vez desde que se adquirió una silla de ruedas en cada participante. En el estudio participaron 6 adultos, 5 entre 40-54 años de edad y 1 caso de 18 años de edad. La metodología fue de tipo cualitativo-descriptivo y la técnica de recolección de información que se utilizó fue observación participante y entrevistas semiestructuradas. Los resultados muestran que el crecimiento personal es la dimensión que se ve transformada por la práctica deportiva, debido a los aprendizajes y nuevos hábitos que se incorporan, asociados también al desarrollo de estrategias para el manejo de la salud.

\section{KEY WORDS}

Psychological Well-Being Disability

Model of Diversity

Sport

\section{ABSTRACT}

This research was conducted in order to analyze the contribution of handball practice adapted to the level of post-training process psychological well-being (BP). BP was analyzed from the six categories raised by Ryff (1989) self-acceptance, positive relations with others, Domino environment, Autonomy, Purpose in life and Personal growth, these categories related to the sport experience since the first time the partakers acquired a wheelchair. The study included 5 adults between 40-54 years, only one case of 18 years. The methodology was qualitativedescriptive and the recollecting information technique used was participant observation and depth interviews. The results show that personal growth is the dimension that is transformed by the sport due to learning and new habits that are incorporated, also associated with the development of strategies for health management.

\section{GLOBAL $\underset{\text { KCADEMCS }}{\text { KNEDGE }}$}




\section{Introducción}

$\mathrm{S}$ egún la Organización Mundial de la Salud, OMS, en el 2011 refiere que en el mundo más de mil millones de personas viven con algún tipo de discapacidad. En Colombia se presenta una tasa de prevalencia de la discapacidad del $12 \%$ de la población total más de 4 millones de personas. El Departamento Administrativo Nacional de Estadística (DANE) en el 2012, estimó un porcentaje aproximado de personas con por lo menos alguna limitación 6,4\% del total de la población, que equivale aproximadamente a 2.632 .255 personas. De acuerdo a estas cifras, por cada 100 colombianos, con limitaciones el 29,3\% tienen limitaciones para caminar o moverse, esto equivale a 770.128 personas, siendo Valle del Cauca una de las regiones con el porcentaje más alto. De esta manera, para esta investigación es de vital importancia conocer los deportes ofrecidos por el Comité Paralímpico Colombiano (CPC) para las limitaciones físicas, los cuales son: Ajedrez, Atletismo, Baloncesto en Silla de Ruedas, Billar, Ciclismo, Levantamiento de Pesas, Natación, Tenis de Campo en Silla de Ruedas, Tenis de Mesa, Tiro Deportivo. De esta manera, encontramos que el Balonmano en Silla de ruedas, es un deporte nuevo en Colombia, que acogerá a personas con ninguna experiencia en el campo del deporte. Por esto mismo es de interés, analizar en esta investigación la contribución de este deporte en personas con diversidad funcional física que lo practican por primera vez.

\section{Revisión de literatura}

\section{Bienestar psicológico}

El concepto bienestar psicológico en la actualidad puede ser abordado desde diversos campos de estudios, específicamente desde la psicología. De esta manera, la psicología positiva ${ }^{1}$ definida por Seligman (1999) inicia sus estudios con tendencia a las variables positivas y preventivas en lugar de los aspectos negativos y patológicos que tradicionalmente se estudian 2. Así, surgieron modelos teóricos, Ryff (1995) propone una perspectiva unificada de estos ${ }^{3}$. Pese a sus numerosas y cambiantes definiciones, en esta investigación la categoría bienestar psicológico (BP)

\footnotetext{
${ }^{1}$ Estudio científico de las experiencias positivas, los rasgos individuales positivos, las instituciones que facilitan su desarrollo y los programas que ayudan a mejorar la calidad de vida de los individuos, mientras previene o reduce la incidencia de la psicopatología (Seligman 2005; Seligman \& Csikszentmihalyi, 2000).

2 (Guerrero \& Vera, 2003; Simonton \&Baumeister, 2005) Citado por Contreras \& Esguerra (2006).

3 Teorías del desarrollo humano óptimo, el funcionamiento mental positivo y las teorías del ciclo vital.
}

será considerada a partir de la composición de seis dimensiones diferenciadas planteadas por (Ryff y Keyes, 1995) nombradas a partir del "Modelo Integrado de Desarrollo Personal" (Integrated Model of Personal Develoment": 1) auto-aceptación; 2) relaciones positivas con otros; 3 ) dominio del entorno; 4) autonomía; 5) propósito en la vida; 6) crecimiento personal (citado en Solano, 2009, P47) las cuales se definirán en los siguientes párrafos:

La auto-aceptación es definida por Ryff (1989) como el criterio más constante del bienestar evidente en las perspectivas previas, es el sentido individual de la aceptación propia. Esto es definido como un rasgo de salud mental, también como una característica de la actualización propia, óptimo funcionamiento y madurez. Teorías del lapso de la vida también enfatizan en aceptación de sí mismo y de la vida pasada. Así, teniendo actitudes positivas hacia uno mismo emerge como una característica central de la función psicológica positiva.

Relaciones positivas con otros, Ryff (1989) define la importancia de la confianza en las relaciones interpersonales. Refiriendo que los sujetos autorrealizados se describen con altos sentimientos de empatía y afecto con otros, siendo capaz de un amor más grande, la amistad y la identificación con los demás. Tener la capacidad y calidad de relación es planteado como un criterio de madurez.

El dominio del entorno, Ryff (1989) lo define como la capacidad del individuo para elegir o crear ambientes adecuados a sus condiciones psíquicas, así como la participación en una esfera importante de actividad fuera de sí mismo, el desarrollo de esperanza de vida, como la capacidad de manipular y controlar entornos complejos. Enfatiza en la capacidad para avanzar en el mundo y cambiar de manera creativa a través de actividades físicas o mentales.

Autonomía, Ryff (1989) la describe a través de cualidades tales como la autodeterminación, la independencia y la regulación de la conducta. La persona en pleno funcionamiento tiene la capacidad de realizar una autoevaluación, mediante la cual no se ve a los demás para su aprobación, pero se evalúa a sí mismo por estándares personales. La persona ya no se aferra a los miedos colectivos, creencias y leyes de las masas. Alude al proceso de volverse hacia adentro, para que la persona obtenga una sensación de libertad desde las normas que rigen la vida cotidiana.

Propósito en la vida, según Ryff (1989) incluyen las creencias que le dan a uno la sensación que hay un propósito y significado a la vida. La definición de bienestar también hace hincapié en una comprensión clara del propósito de la vida, un sentido de direccionalidad y de intencionalidad. Hace referencia a cambiar una variedad de propósitos o metas en la vida, como ser productivo y creativo, o lograr la integración emocional en su vida posterior. Por lo tanto, una persona que 
funciona positivamente tiene objetivos, intenciones y un sentido de dirección, todo lo cual contribuye a la sensación de que la vida es significativa.

Crecimiento personal, Ryff (1989) refiere que no sólo es necesario alcanzar las características anteriores, sino también seguir para desarrollar el propio potencial, para crecer y expandirse. La necesidad de actualizarse uno mismo y darse cuenta de las potencialidades, es fundamental para las perspectivas clínicas en el crecimiento personal. La apertura a la experiencia, por ejemplo, es una característica clave de la persona en pleno funcionamiento. Tal individuo está en desarrollo y puede convertirse continuamente, en lugar de lograr un estado fijo en el que todos los problemas se resuelven. Hace énfasis explícitamente en el crecimiento continuo y la confrontación de nuevos retos o tareas en diferentes períodos de la vida.

El bienestar psicológico es entendido como (...) el resultado de la percepción personal de que la vida se está viviendo bien y adecuadamente, siendo consciente del correcto desarrollo de sus potencialidades (...) (Ryff y Keyes, 1995, citado en Rivas, C., Romero, A., Pérez-Llantada, M. d. C., López de la Llave, A., Pourtau, M., Molina, I., González, J. \& Garcia-Mas, A., 2012. P365) Este modelo de bienestar psicológico se enmarca dentro del desarrollo del potencial humano denominado, bienestar eudaimónico. Esta perspectiva plantea que éste viene determinado por la realización de actividades personalmente expresivas que facilitan la autorrealización a través del cumplimiento de potenciales personales y de la promoción de los objetivos personales (Waterman, 1993). La perspectiva eudaimónica es un proceso activo derivado de la búsqueda y el logro de metas personales (Chatzisarantis \& Hagger, 2008).

El bienestar psicológico como lo indica el modelo de Ryff (1989), es dinámico y multidimensional porque cada una de las categorías de bienestar psicológico está constituida por elementos subjetivos, sociales y psicológicos, además de comportamientos saludables. En este sentido, su planteamiento hace énfasis en que los procesos motivacionales y emocionales son esenciales para comprender la relación que establece un individuo con su medio, y están relacionados con un funcionamiento psicológico saludable. De esta manera, Ryan y Deci (2001) señalan "la estrecha relación existente entre motivación intrínseca y bienestar y, por otra parte, numerosas investigaciones indican la asociación entre emoción y bienestar" (citado en Núñez, L., León, J., González, V., \& Martín-Albo, J. 2011. P225)

Los hallazgos de las investigaciones en torno al tema discapacidad-bienestar psicológico-deporte, han arrojado como tema en común la práctica deportiva como un novedoso proceso de rehabilitación. Desde el 2013, la investigación de J. Medina; A. Chamarro, y E. Parrado, analizan la relación entre el bienestar psicológico y el tipo de práctica deportiva en personas con discapacidad física de origen neurológico. Fernhall B \& colaboradores (citado en Medina, 2012) plantean que se estima que las personas con discapacidad realizan una actividad física moderada y actividades de ocio un 50\% menos que las personas sin discapacidad. A partir de estas apreciaciones Medina \& colaboradores, evaluaron el bienestar psicológico de un total de 160 personas con discapacidad mediante el Índice de Bienestar Psicológico (IBP). Para el análisis los sujetos fueron categorizados en 4 grupos en relación con la práctica de actividad física: rehabilitación, deporte recreación, deporte competición y sedentarios. Los resultados encontrados por los autores muestran que los participantes del grupo de sedentarios presentan significativamente un menor bienestar psicológico para todas las dimensiones del IBP, excepto para el resto de grupos. Por otro lado, los participantes del grupo de deporte de competición presentan valores superiores en las dimensiones de autocontrol y de salud general. En este sentido Galea (citado en Medina, 2012) plantea que la rehabilitación física y la práctica de ejercicio aparecen como mecanismos potentes de mejora de la salud. En conclusión, la práctica de actividad deportiva, especialmente competitiva repercute en un mayor bienestar psicológico en personas con gran discapacidad.

Gore; Uchino, y Holt-Lunstad, 1999 (citado en Rivas, et al., 2012) sugieren que el apoyo social y los recursos personales podían actuar como "amortiguadores" del estrés y del riesgo que éste produce en la salud mental y física de las personas. En este sentido, indican precozmente la necesidad de considerar la "intención" personal como una toma de decisiones que debería tener consecuencias respecto de la práctica saludable de un comportamiento motivado (p.366). En 2012, Leeuwen Van y colaboradores en su investigación encontraron que el tiempo transcurrido desde la lesión puede ser una variable que afecta a la satisfacción vital expresada. Por esto, no es descartable que parte del efecto sea atribuible al hecho de llevar tiempo realizando actividades laborales o de la vida diaria (citado en J. Medina et al., 2013, p.14)

Es importante resaltar que al hablar de bienestar psicológico en población con diversidad funcional este estudio realiza una comprensión del sujeto a partir de una perspectiva social. Es por esto, que la esfera social en las últimas décadas del siglo XX tuvo cambios significativos en los marcos legales $y$ políticos de la discapacidad, a nivel nacional e internacional, dirigidos a promover los derechos de participar en todas las esferas: social, laboral, familiar y afectiva. De acuerdo a esto, el modelo de la diversidad planteado por (Palacios \& Romañach, 2008) parte de una visión basada en los Derechos 
Humanos y se orienta en el campo de la bioética ${ }^{4}$ "una herramienta fundamental para alcanzar la plena dignidad de las personas discriminadas por su diversidad funcional"(p.37) El modelo que denominan social, define las causas de esta diversidad desde la construcción social, ya que este concepto se puede construir a diario, en las relaciones sociales con las personas, entre los grupos sociales, en el tejido social; también en las decisiones personales, en las actitudes que se asumen frente a determinada situación $y$, en la manera que se estructura el entorno físico, social, cultural en el que se desenvuelven estas personas. En este sentido es posible referir que cada uno debe lograr una transformación personal a través de los derechos humanos, donde se recuperan aspectos como la autonomía e independencia. También desde lo social, respecto al estigma de la discapacidad "como personas que no son capaces de hacer nada" esta modificación, permite que la población con diversidad funcional se incluya en la comunidad, y reciban igualdad de oportunidades.

En virtud de lo anterior, el aludido modelo pone a cuestionar la perspectiva que refiere la diversidad funcional como una enfermedad individual, por el contrario es desde lo social que se hace esa construcción, por esto Ferrante en el 2013, fundamentó su investigación en torno al tema discapacidad-cuerpo-deporte, encontrando un conjunto de hábitos que son promovidos a través de la práctica deportiva y que dibujan el cuerpo discapacitado valorado al interior del campo, es decir, con ese conjunto de prácticas se apunta a que ese cuerpo se adapte a unos estándares establecidos y valorados por la sociedad. En este sentido para la autora "los saberes exigidos en la forma de reglas de convivencia constituyen fundamentalmente técnicas del cuerpo que cubren las falencias(...) (no se les reconoce como sujetos, no pueden circulan libremente en la ciudad, no se garantiza el acceso al trabajo y a la educación, se quedan fuera del mercado matrimonial), (...) son conocedores prácticos de que, sin un fuerte grado de permeabilidad individual, el destino que les espera es la muerte social"(P.175). Los resultados de su investigación muestran que los participantes cuando se encuentran dentro del campo $y$, parafraseando a la autora, se promueven reglas de convivencia que infieren una adaptación al medio, y saberes que son prácticos porque promueven la salud y saldan las carencias del proceso de rehabilitación. "La mayoría de ellas son estrategias de atención de la salud que abarcan: el ejercicio de la voluntad, el cuidado de la higiene corporal, el control del peso y la prevención de escaras". (P. 165).

\section{Metodología}

Tipo de estudio. La investigación fue de corte cualitativa- descriptiva (Sandoval, 1996), el muestreo es de casos homogéneos, se recolectó la información a partir de la retrospectiva y significado de los participantes frente a sus experiencias comunes en relación al núcleo temático al que apunta la investigación.

Participantes. En la investigación participaron 6 adultos con diversidad funcional física adquirida. Pertenecientes a la Fundación para el Desarrollo Social y Discapacitados (FUNDESD), organización privada sin ánimo de lucro, perteneciente a un estrato socioeconómico bajo. Este grupo se conformó por 1 mujer, 5 hombres comprendidos en su mayoría en un rango de edad de 40-54 años, y un participante de 18 años de edad. Los diagnósticos médicos principales son (4) sujetos con trauma raquimedular y (2) sujetos con secuelas de poliomielitis. En este sentido, se mencionan los criterios por los cuales estos son los participantes del presente estudio: - Jóvenes-Adultos cuyo nivel de lesión implica la utilización de silla de ruedas para su desplazamiento. - no habían practicado deporte desde que adquirieron la silla de ruedas. que aceptaron participar de manera voluntaria en el estudio y dieron su consentimiento por escrito.

Los nombres de los entrevistados fueron modificados con el fin de garantizar el carácter anónimo y confidencial de las entrevistas, a cada participante se le identifica con el siguiente nombre ficticio: "Ana, Juan, Leo, Hugo, Eric y Luis".

Instrumentos. Se empleó como técnicas de recolección de información, la observación participante y entrevistas semiestructuradas.

Recolección de información. Durante la investigación se llevó a cabo la implementación de la práctica de balonmano en silla de ruedas durante 6 meses. La planificación del programa de entrenamiento de balonmano adaptado se realizó basándose en las capacitaciones ${ }^{5}$ donde se tuvieron en cuenta las características de la población y las actividades posibles a realizar con ésta. Por otro lado basándose en la teoría y metodología del entrenamiento deportivo, se elaboró un macrociclo de entrenamiento que consto de una mesociclo introductorio en el cual se llevó a cabo la iniciación y fundamentación teórico-práctica de Balonmano adaptado por medio de actividades pre-deportivas, y un mesociclo preparatorio donde se trabajaron los componentes de preparación física general y específica, aspectos técnicos, tácticos y psicológicos. Posteriormente los entrenamientos se llevaron a cabo en una cancha que cumplía con los parámetros

\footnotetext{
${ }^{5}$ Curso de actividades físicas para personas con discapacidad física, en el Instituto del deporte, la educación física y la recreación del Valle del Cauca (INDERVALLE, 2013), participación en el I Torneo Panamericano de Balonmano en Silla de Ruedas, en Buenos Aires-Argentina (2014). 
mínimos para la práctica de este deporte, se utilizaron balones tipo softball, conos, therabands, botellas de arena, palos, cronómetro y pito. Las sesiones se ejecutaban 2 veces a la semana, los días martes y jueves con una intensidad de 3 horas, se realizaba calentamiento $\mathrm{y}$ enfriamiento para la prevención de lesiones, realización de actividades físicas y trabajo en equipo, se tuvo en cuenta el proceso de hidratación durante todo el entrenamiento. Los encuentros para las entrevistas se ajustaron después de los seis meses de entrenamiento, en un lugar que cumpliera los requisitos de privacidad y tranquilidad para su aplicación se efectuó dentro de la institución en un salón, en el horario anterior al entreno.

Tabla 1.1. Caracterización sociodemográfica del grupo de participantes

\begin{tabular}{|c|c|c|c|c|c|c|}
\hline $\begin{array}{c}\text { Sujetos } \\
\text { Participantes }\end{array}$ & Edad & Trabajo & $\begin{array}{l}\text { Tiempo de adquisición de } \\
\text { la discapacidad }\end{array}$ & Tipo de discapacidad & $\begin{array}{l}\text { Personas con } \\
\text { quien viven }\end{array}$ & Hijos \\
\hline Ana & 54 & No & 52 años & Secuelas poliomielitis & Hija y nieta & 2 \\
\hline Juan & 42 & Pensionado & 6 años & $\begin{array}{l}\text { Trauma raquimedular } \\
\text { T10 }\end{array}$ & Padres & 2 \\
\hline Leo & 45 & Pensionado & 6 años. & $\begin{array}{c}\text { Trauma raquimedular } \\
\text { T7 }\end{array}$ & Pareja & 1 \\
\hline Hugo & 49 & Independiente & 47 años & Secuelas poliomielitis & $\begin{array}{l}\text { Pareja y } \\
\text { cuñado }\end{array}$ & No \\
\hline Eric & 18 & No & 4 años & $\begin{array}{c}\text { Trauma raquimedular } \\
\text { T7 }\end{array}$ & $\begin{array}{l}\text { Madre y } \\
\text { hermano }\end{array}$ & No \\
\hline Luis & 41 & Pensionado & 19 años & $\begin{array}{c}\text { Trauma raquimedular } \\
\text { T6 }\end{array}$ & Solo & No \\
\hline
\end{tabular}

Fuente: Elaboración propia, 2015.

Procedimiento. Se realizó la presentación del anteproyecto a FUNDESD. Los datos de los participantes se obtuvieron a partir de la información proporcionada por esta Fundación. Se elaboró una base de datos con 20 jóvenes y adultos que se movilizaban por medio de silla de ruedas, posterior a eso se realizó una convocatoria vía telefónica de la cual 15 personas que cumplían con los criterios de inclusión asistieron a una reunión, 6 accedieron a participar voluntariamente en el estudio. La observación participante se realizaba durante el entrenamiento $\mathrm{y}$ al finalizar se tomaba registro de audio de las conversaciones grupales acerca de las dificultades y aspectos positivos durante el entrenamiento ese día, los indicadores que guiaron estas observaciones fueron: dominio del entorno, relaciones positivas con los demás y crecimiento personal de Ryff(1989). La entrevista semiestructurada se aplicó de forma individual y duraron aproximadamente dos horas cada una. Se inició indagando sobre la valoración de bienestar que percibe cada participante en todas las áreas de su vidas: origen de la discapacidad, transformaciones que introdujo la discapacidad en su vida, experiencias asociadas al trabajo y beneficios del deporte, las preguntas fueron orientadas cada una por las categorías de bienestar psicológico propuesta por Ryff (1989). Las entrevistas fueron grabadas en audio y transcritas para su posterior codificación. El proceso de interpretación y análisis de los resultados se realizaron transcripciones literales, se seleccionaron los relatos más significativos de cada categoría, con un microanálisis línea por línea. Cada categoría en la investigación aporta de manera significativa en las dinámicas que se generan en una práctica deportiva, se analizaron mediante una codificación en base a la teoría de bienestar psicológico compuesto por las seis dimensiones: Autoaceptación, Relaciones positivas con los demás, Dominio del entorno, Autonomía, Propósito en la vida y Crecimiento personal propuesta por Ryff(1989).

Aspectos éticos. Las personas participaron voluntariamente en el proceso. Se realizó un consentimiento informado, donde se explicó a los sujetos los detalles de la investigación y se les solicitó el asentimiento para su inclusión en esta. Se realizó énfasis en la confidencialidad, indicando que la información recolectada no incluiría nombres propios que afecten el buen nombre de los informantes. Finalmente el derecho sobre la información, en el cual los participantes conocieron los procesos llevados a cabo y los resultados.

\section{Resultados}

A continuación, se presentan los resultados. La tabla 1.2, delinea la categoría, definición y el discurso de apoyo. 
Tabla 1.2: Categorías que reflejan las dimensiones del bienestar psicológico

\begin{tabular}{|c|c|c|}
\hline Categoría & Definición & Discurso de apoyo \\
\hline Autoconcepto & $\begin{array}{l}\text { Es el sentido } \\
\text { individual de la } \\
\text { aceptación } \\
\text { propia y de la } \\
\text { vida pasada, } \\
\text { teniendo } \\
\text { actitudes } \\
\text { positivas hacia } \\
\text { uno mismo }\end{array}$ & $\begin{array}{l}\text { "mis días los empecé a ser más variables, voy al nuevo latir a hacer deporte, otro día } \\
\text { voy a visitar a mis hermanos, a dar vueltas por la ciudad, también a jugar parques o } \\
\text { dominó. Ya no me gusta quedarme encerrado porque eso conlleva a realizar } \\
\text { actividades malas, siempre busco algo para distraerme". } \\
\text { "Desde muy pequeña mi mamá me enseñó a hacer todo lo de la casa, me trato como } \\
\text { una persona normal". } \\
\text { "Mi reacción en esos momentos fue llorar y llorar y pensar que se me había } \\
\text { arruinado la vida. Y desde ese día, desde el primer momento que me pegaron esos } \\
\text { tiros empezó mi sufrimiento. Yo mantenía encerrado solo salía a citas médicas". } \\
\text { "yo me volví grosero, me volví amargado, la depresión me cogió. Uno escucha que la } \\
\text { gente dice que la gente en silla de ruedas no sirve, y por eso mismo se terminó la } \\
\text { relación con mi ex mujer por esto". }\end{array}$ \\
\hline $\begin{array}{l}\text { Relaciones } \\
\text { positivas } \\
\text { otros }\end{array}$ & $\begin{array}{l}\text { Confianza en las } \\
\text { relaciones } \\
\text { interpersonales } \\
\text { teniendo la } \\
\text { capacidad } \quad \text { y } \\
\text { calidad de } \\
\text { relación. }\end{array}$ & $\begin{array}{l}\text { "No confió en nadie por muchas cosas que me han pasado. Entonces he vivido en el } \\
\text { anonimato prácticamente" } \\
\text { "Me he sentido más apoyada por mis amigas que por mis mismos familiares de mi } \\
\text { misma casa". } \\
\text { "Cuando fui los primeros días al entreno veía que muchos tenían una sonrisa y yo } \\
\text { muy malgeniado, reflexione y con ayuda de todos esto cambio mi actitud hacia la } \\
\text { discapacidad empezó a cambiar". }\end{array}$ \\
\hline $\begin{array}{l}\text { Dominio } \\
\text { entorno }\end{array}$ & $\begin{array}{l}\text { Capacidad para } \\
\text { crear o elegir } \\
\text { ambientes } \\
\text { adecuados a su } \\
\text { condición } \\
\text { psíquica, la } \\
\text { participación } \\
\text { capacidad } \\
\text { manipular } \\
\text { controlar } \\
\text { entornos } \\
\text { complejos. }\end{array}$ & $\begin{array}{l}\text { "Uno depender de otro es muy berraco, si uno se orina y que no lo limpien, es } \\
\text { berraco". } \\
\text { "La terapeuta me daba ideas y así se me hacía más fácil, después me hacía pruebas } \\
\text { para ver que en realidad sí podía hacerlo. Me enseñó a independizarme, a vestirme, } \\
\text { cambiarme de ropa". } \\
\text { "Yo vivo del rebusque, por ejemplo en semana santa vendo sahumerio, artículos religiosos, y } \\
\text { con mi esposa tenemos una chazita donde vendemos dulces". } \\
\text { "Mi pensión la uso en mis servicios, yo pago arriendo, la comida, y también la comida } \\
\text { de mis hijos, en eso la distribuyo". } \\
\text { "Aquí mismo en la fundación los martes en la mañana estoy haciendo un curso de } \\
\text { sistemas, invite a mi hermana } \\
\text { "Todos mis días son iguales. A diferencia de los martes y viernes que entreno balonmano". } \\
\text { "Yo me dedico a los oficios de la casa, lavo los platos cuando los dejan sucios, cocino, } \\
\text { ese es mi trabajo y me siento bien porque me dan un sitio y un papel en la casa". }\end{array}$ \\
\hline Autonomía & $\begin{array}{l}\text { Cualidades la } \\
\text { como la } \\
\text { autodeterminac } \\
\text { ión, la } \\
\text { independencia y } \\
\text { regulación de la } \\
\text { conducta. }\end{array}$ & $\begin{array}{l}\text { "Poco a poco fui aprendiendo a pasarme de la cama a la silla, a cocinar,". } \\
\text { "Mi hijo alcanzó el bachillerato. Mi hija ahora es profesional, aunque ella misma } \\
\text { pago sus estudios. Yo era papá y mamá al mismo tiempo. Luche para sacarlos } \\
\text { adelante, y ellos no pueden decir que mi mamá nos regaló porque no fue así, siempre } \\
\text { estuvieron a mi lado, en los peores momentos." } \\
\text { "Me quedo aterrada como mis compañeros se van a rodar por la ciudad, y por eso } \\
\text { ahora no me da miedo coger un bus. Porque tengo que darme la seguridad. Si ellos } \\
\text { pueden, yo también" } \\
\text { "muchas personas les daba pesar de mi". } \\
\text { "cuando vivía con mi mujer ella me lo hacía todo, mi señora me ayudaba a bañarme, } \\
\text { a vestirme". } \\
\text { "No tengo nada mío. A mí me mantiene mi abuela, ella me da todo. Lo más duro de } \\
\text { haber quedado así es que no puedo trabajar". } \\
\text { "Me dio rabia, a lo primero ellos mantenían ahí pendientes de mí, de mi cepillo, de pasarme } \\
\text { todo, pero después del tiempo ya me dejaban ahí tirado y todo mundo hacía lo suyo" } \\
\text { "Yo no uso pañales, sondas, pero no es porque no quieran sino que su movilidad no } \\
\text { les da para eso. Por el contrario yo sí puedo defenderme ante esos puntos, que es la } \\
\text { independencia". }\end{array}$ \\
\hline Propós & $\begin{array}{l}\text { Creencias que } \\
\text { dan sensación } \\
\text { que hay un } \\
\text { propósito y } \\
\text { significado de } \\
\text { vida, teniendo } \\
\text { sentido de } \\
\text { direccionalidad y } \\
\text { de } \\
\text { intencionalidad }\end{array}$ & $\begin{array}{l}\text { "Me mandaron para la casa y vi una silla de ruedas, además que mi mamá tenía que } \\
\text { vestirme que me ponían pañal, cuando eso pasó empecé a entrar en la depresión y } \\
\text { me intente suicidar a los dos meses de haber quedado así". } \\
\text { "Cuando me dispararon yo sentí que alguien me empujo y no me alcanzo a entrar en } \\
\text { la cabeza, si no es por eso yo hubiera caído de una muerto. Aunque yo mire y no } \\
\text { había nadie. Yo ahora me pongo a pensar y veo y creo que fue Dios. Me dio otra } \\
\text { oportunidad de vida. Yo creo en él, a mi manera, a mí me criaron católico" } \\
\text { "Ahora adulto me gusta mucho hacer cursos y capacitaciones, aprender nuevas } \\
\text { cosas sobre todo que tengan que ver con esto de la discapacidad". } \\
\text { "Yo quiero terminar mi estudio y hacer una carrera o un curso". } \\
\text { "Mis hijos, verlos salir adelante, siendo unas personas estudiadas, es lo que me motiva } \\
\text { para salir adelante. Quiero estar vivo y presente para ver lo bueno que ellos hagan por } \\
\text { sus vidas. Me sentí muy orgulloso cuando mi hija se graduó este año de bachillerato" }\end{array}$ \\
\hline
\end{tabular}




\begin{tabular}{|c|c|c|}
\hline Categoría & Definición & Discurso de apoyo \\
\hline $\begin{array}{l}\text { Crecimiento } \\
\text { personal }\end{array}$ & $\begin{array}{l}\text { Desarrollar el } \\
\text { propio potencial } \\
\text { para crecer y } \\
\text { expandirse, } \\
\text { teniendo } \\
\text { apertura a la } \\
\text { experiencia }\end{array}$ & $\begin{array}{l}\text { "He tenido que aprender a manejar la silla de ruedas deportiva, es duro porque no } \\
\text { tiene frenos, pero a través de la práctica he podido manejarla bien, puedo girar y } \\
\text { rodar en velocidad, es un logro para mí". } \\
\text { "El deporte y la fundación me ha aportado sobretodo amigos, he conocido personas } \\
\text { muy humanitarias, otra de las cosas es que mi mujer y mi hija pueden venir a } \\
\text { acompañarme entonces compartimos otros espacios". } \\
\text { "Yo uso los óvulos vaginales, ya pasaron } 19 \text { años y las escaras ya no me dan, y si son } \\
\text { muy leves y las sé tratar, entonces no las dejo hacer grandes. Yo mismo me hago mis } \\
\text { tratamientos". } \\
\text { "Alguno tiene esparadrapo se me pelaron dos dedos". Leo responde: "Los } \\
\text { esparadrapos los usan las niñas". Juan: "suenan carcajadas y prosiguen el } \\
\text { entrenamiento". [Extracto de observación participante, } 15 \text { octubre de 2014]. } \\
\text { "He bajado } 40 \text { kilos, pues junto con la dieta y movilizarme rodando". } \\
\text { "Aspiro a que salgamos del país a representarloy hagamos una buena obra. }\end{array}$ \\
\hline
\end{tabular}

Fuente: Elaboración propia, 2015.

Respecto al Autoconcepto atribuido por los participantes, tenemos que su importancia está arraigada a través de la aceptación de ellos mismos y de la vida pasada, primando esto sobre el factor bienestar. Se encontró que prevalece la importancia de sobreponerse y superar la situación vivida. Ante esto, Ryff (1989) refiere que para las personas que poseen una aceptación de sí mismos y su vida pasada tienen un óptimo funcionamiento, una capacidad de auto actualización y por lo tanto se consideran personas con madurez (p.1060), en este sentido los participantes Ana y Hugo son los que refieren una mayor aceptación, enfatizando que la adquisición de la poliomielitis fue a corta edad, y su entorno familiar facilito los recursos necesarios que permitieron que se desenvolvieran y desarrollaran habilidades sin sobreponer una condición biológica. Contrariamente, se encontró que los participantes Juan, Leo, Eric y Luis no se sienten satisfechos consigo mismos y refieren una cierta decepción de lo que ha ocurrido en su vida pasada, se vivenció un proceso de negación manifestado a través del llanto, la tendencia al encierro, las palabras groseras, el malgenio, la indiferencia ante la situación de la vida, y los cambios de estado de ánimo, por la pérdida inicial de la independencia $y$ un factor de preocupación por el uso de silla de ruedas como una cualidad nueva que pertenece a su corporalidad. Ante esto, Ryff (1989), menciona que una característica principal de salud mental es aceptar la vida pasada, y se encontró por el contrario que los participantes quienes aún no aceptan su vida pasada, y no enfatizan su vida en actitudes positivas frente a sí mismos, su énfasis ha estado en otras características como la autonomía y propósito de vida que ha permitido contribuir, como ellos lo expresan, a sentir un bienestar.

De esta manera, al indagar sobre lo que contribuye la práctica deportiva, se encontró que los participantes con diagnóstico de trauma raquimedular, realizaron un proceso de ajuste, al cambiar su hábitos, lo que conllevo a que salieran de sus casas, y llegaran al espacio de práctica, y en Ana, Hugo, también se movilizo la característica de pensar en sí mismo. Por consiguiente, la actividad física aportó como un elemento movilizador de una manera más significativa al grupo de participantes que aún no han aceptado su discapacidad, encontrando que entre menor tiempo lleva la lesión, menor es la aceptación. Este tiempo de acuerdo a la experiencia subjetiva de cada participante esta entre los 5 a los 15 años lograr aceptar la situación. Siendo así, el grupo de participantes con mayor aceptación son aquellos que tienen más años de su lesión. Esto, relacionado con el estudio de Leeuwen Van y colaboradores (2012) quienes hallaron que de acuerdo al tiempo transcurrido de la lesión se ve afectada la satisfacción vital, y que el practicar una actividad puede ser efecto para esta misma.

En la categoria Relaciones positivas con otros, los participantes refieren que su bienestar en términos positivos está en las relaciones de confianza más fuertes, que se encuentran en el núcleo familiar más cercano y donde refieren que el progreso en el proceso de adaptación de su diagnóstico se dio gracias a este apoyo. La mayoría de los participantes refirieron tener mayor apertura hacia las personas de quien han mas recibido ayuda, para uno son los familiares y solo en el caso de Ana, son sus amigas. Por el contrario, se encontraron indicadores bajos de bienestar, todos los participantes refieren una incomodidad al ser parte de reuniones y grupos sociales, esto se debe al establecimiento de una rutina que se reduce del centro de rehabilitación a la casa, del trabajo a la casa, de citas médicas a la casa, una rutina en que poco interactúan con los demás. Se encontró que esta dificultad para un contacto inicial, tener apertura y cercanía con un otro, está asociado a experiencias negativas previas que han tenido con su entorno socio-familiar y han generado la pérdida de confianza, específicamente a nivel de relaciones interpersonales, vivenciaron estas situaciones percibidas como discriminación: burlas, malos tratos, engaño, separación que contribuyeron a la perdida de la apertura. Se infiere que las relaciones de este tipo son un retroceso para el proceso de habilitación, así pues los indicadores bajos en las 
relaciones positivas con otros en los participantes está ligado a la evitación de relaciones que aumenten su malestar psíquico.

Así mismo se encontró que en el ámbito de la práctica deportiva los participantes tienen la actitud de abrirse a nuevas formas de relacionarse, debido a las dinámicas grupales, que implicaban la cercanía y la capacidad de relación, posterior al proceso de entrenamiento todos los participantes refieren que al sentirse parte de grupos sociales en los que encuentran apoyo, un ambiente que facilita el compartir experiencias asociadas a su diagnóstico, se aduce la construcción de un sentido de pertenencia grupal que se logra a través de la interacción, y reforzaría además la autoestima de los participantes, quienes perciben que sus propias experiencias y relatos pueden convertirse en una contribución positiva para el bienestar de sus pares, estos resultados se encuentran en relación al estudio de Palacios y Romañach (2008). En síntesis, surgen dos beneficios clave asociados a la práctica deportiva, son: el sentido de pertenencia a un grupo en que ellos son actores activos, y el incremento de la autoestima al percibirse a sí mismos como agentes en el bienestar de otros.

En la categoría Dominio del entorno, se logró identificar que los indicadores bajos de bienestar, se evidencian en los discursos de Juan, Leo, Eric y Luis porque hay recurrencia en la queja respecto a actividades específicas de su cotidianidad que consideraron se vieron afectadas y los puso en una posición de incapacidad para controlar su entorno externo, en el inicio del proceso de rehabilitación, en relación al aseo personal, ya que los cuidadores eran quienes realizaban estas actividades que anteriormente estos controlaban, la limpieza de esfínteres, el baño, control de horarios para el medicamento, la alimentación. También durante el periodo de adaptación se encontró que la dificultad, refieren incapacidad para ponerse ellos mismos su propia ropa, pasarse de la silla a la cama, bañarse solo, experiencias que significaron desesperanza y desesperación.

En esta línea quienes contribuyeron a que los participantes avanzaran en este aspecto fueron las fisioterapeutas, juega un papel importante, porque son quienes exigían sobrepasar la dificultad para que el control de las actividades externas mejorará. Estos participantes, después de adquirir la discapacidad tenían la capacidad de elegir y crear contextos adecuados a sus necesidades y valores personales, que en su caso corresponden a la búsqueda de oportunidades laborales para cubrir las necesidades básicas propias y la de su familia. En este sentido, se identificó que en el caso de Hugo y Ana eran trabajadores informales, debido a que el sector formal no genera empleos específicos que permitan a la población con diversidad funcional física trabajar, es una variable que afectó considerablemente el bienestar psicológico de los participantes, encontrando que son estos, quienes refieren que la falta de trabajo o de un ingreso económico fijo afecta su bienestar porque los pone en una posición de dependencia frente a los demás, o en otros casos porque no pueden sostener a sus familias.

En este estudio se encontró que la capacidad de avanzar fue a través de la práctica de balonmano, donde todos los participantes tienen la capacidad de manejar entornos complejos, una característica importante como lo dice Ryff (1989) el sentido de dominio del entorno $y$, se ve reflejado en la competencia en la gestión de la práctica deportiva, y está asociada a aspectos del orden de lo vivencial dentro de la misma, como lo es: amarrarse a la silla correctamente, hidratarse, usar la ropa adecuada, movilizarse sin acompañamiento en la ciudad, manejo de la silla de ruedas deportiva, resistencia física al realizar las actividades de entrenamiento físico, todo lo que contribuye a que controlan una amplia gama de actividades externas que están asociadas a controlar aspectos de su cotidianidad, conseguir un trabajo, a practicar un deporte y en el caso de Ana y Leo, al rol de padres. Para explicar lo anterior, es pertinente resaltar los resultados de la investigación de Gore y Holt-Lunstad, citado en Rivas et al., 2012) que aducen la necesidad de considerar la intención personal como una toma de decisiones que tiene consecuencias respecto de la práctica saludable de un comportamiento motivado. En este sentido, se reconoce que la decisión de controlar actividades externas e internas depende subjetivamente de cada participante, es decir que el desarrollo de la voluntad propia se moviliza, porque estas elecciones son de carácter subjetivo. Podríamos pensar que los participantes desarrollan un dominio de su entorno en la práctica deportiva gracias a que se asumen y se ven a sí mismos como sujetos con capacidad de controlar y enfrentar las situaciones que se le presentan a diario.

En la Autonomía, se identificó que surge en los participantes la dependencia como consecuencia principal de la lesión, tras el inicio de su recuperación, el temor surge por la pérdida de la movilidad y a la no posibilidad de realizar las mismas actividades que anteriormente se realizaban de manera autónoma. Estas nuevas dinámicas de relación se convierten en un desafío y una característica con dos perspectivas. Por un lado, se encuentra que s la independencia es lo primero que quiere recuperarse después de tener una lesión medular, de esta manera los participantes inician una serie de prácticas de la vida diaria como: cocinar, comer solo, bañarse solo, vestirse solo, limpiar su casa solo, un "solo" que se "re-traducen, discursivamente, en la dicotomía cuidarse/no cuidarse, expresando una visión voluntarista de superación de la discapacidad". (Ferrante, 2013, p.161).

No obstante, a pesar de que la dependencia para todos los participantes es el principal objetivo, dos de los participantes significaron que el soporte recibido debía ser incondicional y en todos los momentos de su vida. De esta manera, la capacidad 
de autoevaluación planteada por Ryff (1989) está ausente y se vivencia la actitud de aprobación de los demás específicamente de su cuidador(a) para actuar. Encontrando pues, que aunque desean ser independientes, el sentirse acompañados, cuidados y apoyados, es lo que quisieran conservar pero que en algún momento finaliza, específicamente cuando se incluyen en una práctica deportiva. Ferrante (2013) abordó lo que surge con las personas con discapacidad física dentro de un contexto deportivo, encontrando que se promueve dentro de este el pensamiento de "no existe el no puedo, sino el no quiero". Dicha moral se instaurará a través de un "proceso mimético, en el cual, cuerpo a cuerpo, se transmiten una serie de hábitos corporales que entrenan a la persona con discapacidad con el fin de potenciar sus capacidades remanentes y maximizar su calidad de vida" (p.165). Así, cuando se habla del proceso mimético, se hace referencia al ser capaz de resistir las presiones sociales, a pensar en el "sí puedo". Los participantes consideran la autonomía como un valor importante dentro de su recuperación y puede ser optativo como lo evidenciamos anteriormente, en el campo deportivo se convierte en una regla, lo cual puede aducirse como una ambivalencia, ya que depende del contexto en donde se encuentra la persona así mismo este podrá desplegar cualidades como la autodeterminación, la independencia y la regulación de su conducta como es referido por Ryff (1989).

Respecto al Propósito en la vida, resulta pertinente mencionar la creencia como un valor emergente en esta investigación, considerado vital para asumir y dar un lugar a la discapacidad. Pues bien, creencia significa tener la percepción de que existe un propósito y significado en la vida, de esta manera una persona que goce de bienestar tendrá unos objetivos, intenciones y un sentido de dirección, que contribuye a la sensación de que la vida es significativa (Ryff, 1989), los participantes consideran que la creencia está relacionada a un ser superior, denominado como Dios; es precisamente la fe, lo que implicó re significar el hecho de haber adquirido una discapacidad ya que significan la situación, como una salvación ante algo peor o ante la muerte. Aunque esta condición implicó un desgaste psicológico y emocional en todos los participantes durante un tiempo muy prolongado.

De acuerdo a lo anterior, ese desgaste psicológico y emocional tuvo un impacto de acuerdo al momento actual de su vida. Se encontró que los cuatro participantes con diagnóstico de trauma raquimedular significaron el no sentir las piernas como un evento traumático que dio lugar a expresar deseos de muerte con plan estructurado, estos participantes que pensaron en quitarse la vida, llegaron al acto entre una y dos veces. De acuerdo a esto, la investigación de (Chatzisarantis \& Hagger, 2008) corrobora que es en un proceso activo derivado de la búsqueda y el logro de metas personales que los sujetos pueden salir de ese estado emocional, para posteriormente realizar actividades que estén en pro del bienestar.

En cuanto al Crecimiento personal, se encontró que el significado que tiene para los participantes iniciar una práctica deportiva es de alta importancia, ya que refieren que esto permite salirse de su rutina. En este sentido, esta actividad permite un desarrollo del propio potencial, de acuerdo a la experiencia en los participantes se identificó la percepción de mejora en sí mismo y en su comportamiento a través del tiempo de práctica: ya que es aquí donde el aprender el manejo de la silla de ruedas deportiva se convierte en un reto, puesto que al inicio todos los participantes se quejaban de lo pesada, lo grande y lo difícil porque no tiene frenos, a diferencia de la silla de ruedas clínica la cual usan permanentemente, actualmente aducen que es un logro, manejarla bien, aspecto que se ve reflejado en la velocidad con la que recorren la cancha, los giros, y el manejo simultáneo de ir rodando con una mano la silla y con la otra ir driblando el balón. Esta experiencia relatada, permite que los participantes se vean a sí mismos en expansión de habilidades. En esta línea, el estar abierto a nuevas experiencias refiere un funcionamiento psicológico saludable, que se ve reflejado en las frases dichas por estos participantes, "tengo ganas de vivir" "estar con mis hijos" "competir internacionalmente en este nuevo deporte". Se entiende entonces, que el iniciar una práctica deportiva constituye para los participantes una manera de satisfacer su vida al aprender nuevas cosas, al sentirse independientes y valerosos de sí mismos.

Se apuntó a indagar las situaciones que generan crecimiento personal en torno a la elección de practicar un deporte. Ante ello, el cuidado y la prevención de escaras es uno de los problemas más frecuentes para los usuarios de silla de ruedas, ya que se generan por la falta de movimiento al estar en una misma posición y no tener el almohadón anti-úlcera. Así, se puede aducir que dicho conocimiento por Luis es un aprendizaje que logró por sus propios medios y que adicionalmente es transmitido cuando un compañero tiene una escara o la manera de evitarla. Pues bien, depende del cuidado del sujeto tenga por sí mismo, cambiar de posición continuamente, usar su almohadón todo el tiempo, bañarse después de los entrenos, hidratarse correctamente, aquel que no tenga aquellos cuidados podrá tener una escara, y los participantes en la práctica deportiva lo refieren como un descuido. Los participantes deben revisar cada día la ausencia de escara, ya que si se tiene presente no puede realizar actividad física con esto. Por esto, se logra desarrollar el propio potencial, reconocido a través del autoconocimiento y eficacia.

Los participantes expresan el control de peso como norma, es indispensable para mejorar la 
condición de salud y el rendimiento deportivo. Ante ello, Hugo, Leo, Juan y Luis refieren en su discurso tener una sensación de continuo desarrollo. La participación de cuatro de los participantes en el I Torneo Panamericano de Handball en silla de ruedas, significó una experiencia única, denominada como la más grande vivida, ya que cómo lo refieren los participantes la actividad deportiva fue algo que no se realizaba cuando eran caminantes, lo significan como un logro y el desarrollo del sentido de competencia. Esto fue posible gracias a que se movilizaron a participar, se movieron de su zona de confort, y desde que están en el grupo se abren a nuevas experiencias que aportan nuevos referentes para enfrentar su situación.

Es pertinente resaltar lo que Ferrante (2013) aduce frente a la relación deporte y discapacidad, cuando señala que a través del juego se adquieren saberes prácticos que hacen que se construyan estrategias de atención primaria de la salud, difusión de derechos socialmente reconocidos y disminuir los elementos trágicos que ahondan en la discapacidad (p.175). Otra de las dimensiones abordada en cuanto al crecimiento personal por (Ryff, 1989) arroja que la apertura a la experiencia hace parte de una persona que está en pleno funcionamiento. Lo anterior, se puede corroborar, en la observación participante de los entrenamientos, a través de la realización de nuevas tareas que en esta investigación se orientan al aprendizaje no solo de técnicas deportivas en balonmano, sino en estrategias de atención relacionadas al mejoramiento de la salud, como lo son: la prevención y el manejo de escaras, los hábitos saludables de la vida diaria como la alimentación, hidratación, vestuario adecuado para practicar un deporte, el control de peso y esfínteres, el mostrarse resistente e independiente.

\section{Conclusiones}

Los datos obtenidos en el presente trabajo permiten concluir que la práctica de balonmano adaptado está asociada al bienestar psicológico. Los resultados arrojan que la categoría de crecimiento personal se ve transformada en todos los participantes, debido a los aprendizajes y nuevos hábitos que se incorporan y que están asociados también al desarrollo de estrategias para el manejo de la salud. A través del juego se adquiere un quehacer, que anteriormente había estado anulado por una condición de encierro, sufrimiento y estrés que finaliza en pérdida de aquello que se constituye como motor para continuar luchando: su bienestar. Es por esto que facilitar un espacio deportivo en donde se reconozca al sujeto no sólo como un aprendiz, sino como un ser humano integrado, es de vital importancia. En el presente estudio se encontró que todas las categorías del bienestar psicológico se interrelacionan entre sí, encontrando que la diversidad funcional física es una condición que viene marcada en la subjetividad de los participantes y se hace visible dentro del contexto deportivo, mediado por una historia de vida que especialmente está atravesada por la vivencia pre y post de un proceso de rehabilitación.

Con el fin de dar respuesta a la pregunta que orienta esta investigación, la cual está dirigida a identificar la contribución en el bienestar psicológico por la práctica de balonmano, se encontró en la interrelación que, el pensar en sí mismo es un proceso que está ligado a la autoaceptación y se movilizó en tres de los participantes, y está asociado a realizar cosas que antes no se hacían por la no aceptación a la vida pasada. En esta línea en las relaciones positivas con los demás, el contexto deportivo indujo a través de las dinámicas grupales a que la actitud fuera abierta, calidad y contribuyó para que tres de los participantes se abrieran a las relaciones interpersonales. En el dominio del entorno, se encontró que la elección de practicar un deporte es de carácter subjetivo y significó para todos los participantes controlar una amplia gama de actividades externas, las cuales están asociadas a aspectos del orden de lo vivencial. En cuanto a la autonomía, se encontró que todos los participantes significan que aquellos que requieren de más controles son aquellas personas con más dificultades para la autonomía, es decir, que la independencia que significan los participantes está arraigada al control de su salud. Así mismo, el propósito de vida a través de la vinculación a la fundación, produjo en los participantes tener tiempo disponible para realizar actividades que generan placer en sí mismos, que estuvo atravesada por unos deseos del orden de lo subjetivo para cambiar esa situación, orientada a culminar su escolaridad, realizar cursos y competir internacionalmente en el deporte. En conclusión, según lo expresado por los participantes de acuerdo al bienestar global, los resultados indican que los sujetos que inician una práctica deportiva expresan un mayor bienestar psicológico, lo anterior refiere los aportes en cada dimensión por realizar la práctica de balonmano; así, se encuentra una relación con la investigación de Medina, Chamarro, Y Parrado (2013) quienes arrojan que los sujetos con la idea que su estilo de vida es activo y que incorpora actividad física y deportiva contribuye a una mayor calidad de vida y bienestar, incluso en sujetos con discapacidad grave.

Si bien el presente estudio apuntó a explorar sobre la contribución por una práctica deportiva, se encontró que los sujetos participantes evidenciaron diferencias no significativas por practicar un deporte en función del trabajo y las relaciones de pareja, por el contrario el hallazgo refiere que el malestar psicológico aumenta por estos dos factores. Por eso, para ahondar en estos estudios, se sugiere continuar con investigaciones sobre el tema, pensando en la integración del modelo de bienestar 
psicológico junto a constructos como la sexualidad, la autoestima, el proyecto de vida, la relación de pareja, la calidad de vida y la corporalidad. Teniendo en cuenta que estos encuentran relacionados con una condición particular, con la historia de vida de los sujetos, las cuales se encuentran en diferentes fases. Se considera la importancia de que próximas investigaciones también indaguen sobre la trayectoria del tiempo transcurrido a partir de la lesión, ¿subjetivamente que implica iniciar una discapacidad adquirida? ¿Cuánto tiempo toma adaptarse a la discapacidad? realizar estudios de casos donde se explore esa cierta trayectoria, con el fin de comprender como subjetivamente ha significado la experiencia de la discapacidad, aclarando que cada vivencia es particular, según lo encontrado en el presente estudio. Además, importante resaltar que para próximas investigaciones se indague también con una población más extensa con el fin de que se consoliden los hallazgos encontrados en esta investigación.

En el presente estudio hay un solo caso donde la participante es una mujer. El caso de Ana, se evidencia a través de su discurso inicial una disminución de bienestar en la mayoría de las categorías que se configuraba en el imaginario del "no poder", después de adquirir la práctica deportiva la contribución en esta participante se dio a través del empoderamiento, al abrirse a las relaciones personales, al aumento en el control de su medio, al aprender a manejar la silla deportiva, a salir a rodar por la calle, ampliar sus metas en cuanto al estudio, el cuidado de su apariencia física, encontrando que la interrelación de cada categoría permite como resultado una mejora a nivel de su bienestar psicológico. De acuerdo a que la práctica la realizó junto a hombres, se sugiere que otros estudios exploren sobre este aspecto, si este es lugar de la mujer ¿cuál es del hombre? ¿Cómo se inviste la feminidad de una mujer con diversidad funcional física adquirida en el espacio deportivo? ¿Cómo les permite afirmarse y ponerse en un lugar distinto frente al mundo? Esto se considera importante porque se aduce que quizás la práctica deportiva no cumple un mismo efecto en la mujer que en el hombre.

Para finalizar, este estudio invita inicialmente a una ampliación del panorama de la Psicología del Deporte, pues si bien es cierto desde este campo se ha indagado desde su tradición en aspectos relacionados al rendimiento deportivo y específicamente en población convencional. Sin embargo, no es muy común que desde este campo se indague con población con diversidad funcional en los aspectos subjetivos que surgen al ingresar a un contexto deportivo. Se invita a la psicología a pensarse otros espacios de intervención que pueden surgir en Fundaciones, instituciones y con poblaciones en estratos socioeconómicos vulnerados, con el fin de promover programas, espacios de psicoeducación, escucha e integración, desde investigaciones cualitativas con el fin de reconocer el impacto del contexto deportivo en la subjetividad de cada sujeto.

\section{Agradecimientos}

Se agradece inicialmente a la Fundación FUNDESD por la oportunidad brindada de implementar el proyecto. A su director, Elías Doncel, quien me permitió hacer parte de ello. En especial, agradecimiento a los integrantes de la Fundación por su cálido recibimiento, por su disposición y disponibilidad para el desarrollo de esta investigación, esencialmente, por permitirme conocer un gran segmento de sus vidas. 


\section{Referencias}

Contreras, F; \& Esguerra, G. (2006) Psicología positiva: una nueva perspectiva en psicología. Red de Revistas Científicas de América Latina, el Caribe, España y Portugal, 2(2), 311-319, Recuperado el día de diciembre del 2014 de: http://www.redalyc.orgбarticulo.oa?id=67920210

Dane. (2012) CENSO. Recuperado el día 18 de enero del 2015 de: http://www.dane.gov.co/index.php/esp/poblacion-y-registros-vitales/discapacidad/119demograficas/discapacidad/2848-discapacidad-por-departamentos

Ferrante, C; \& Ferreira, M. (2011) Cuerpo y habitus: el marco estructural de la experiencia de la discapacidad. Revista sociológica de pensamiento crítico, 5(2), 85-101. Recuperado en febrero de 2014 de: http://webcache.googleusercontent.com/search?q=cache:http:// scholarlyexchange.org/ojs/index.php/InterSoc/article/download/8888/6229

- (2012). Luchas simbólicas en la definición del cuerpo discapacitado legítimo en el origen e institucionalización del campo del deporte adaptado de la Ciudad de Buenos Aires, Argentina. Revista Latinoamericana de Estudios sobre Cuerpos, Emociones y Sociedad, 9, 3851. Recuperado de:

- (2013). Cuerpo, deporte y discapacidad motriz en la Ciudad de Buenos Aires. Tensiones entre la reproducción y el cuestionamiento a la dominación, Revista Española de Discapacidad, 1, 159-178.

Hagger, M; \& Chatzisarantis, N. (2008) Self-determination Theory and the psychology of exercise. International Review of Sport and Exercise Psychology. 1(1), 79-103. DOI: $10.1080 / 17509840701827437$

Leeuwen, C; Post, M; Woude, L; Groot, S; Smit, C; Kuppevelt , D; \& Lindeman, E. (2012) Changes in life satisfaction in persons with spinal cord injury during and after inpatient rehabilitation: adaptation or measurement bias. Qual Life Res, 21(1), 499-1508. Recuperado el día 26 de junio del 2015 de: http://link.springer.com/article/10.1007\%2Fs11136-011-0073-7

Medina, J. Chamarro, \& E. Parrado (2013) Efecto del deporte en el bienestar psicológico de las personas con gran discapacidad física de origen neurológico. Revista Rehabilitación, 47(1), 1-10. Recuperado el de septiembre de 2013 de: http://dx.doi.org/10.101zo/j.rh.2012.10.006

Molero, F; Ferrero, S; García, C; \& Fernández, I. (2013) La relación entre la discriminación percibida y el balance afectivo en personas con discapacidad física: el papel mediador del dominio del entorno. Acta colombiana de psicología. 16(1). 35-42. Recuperado de: http://portalweb.ucatolica.edu.co/easyWeb2/files/23_11621_v16-n1art3.\%2016\%20No1.pdf

Núñez, L., León, J., González, V., \& Martín-Albo, J. (2011). Propuesta de un modelo explicativo del bienestar psicológico en el contexto deportivo. (Spanish). Revista De Psicología Del Deporte, 20(1), 223-242.

Solano, A. (2009) El bienestar psicológico: cuatro décadas de progreso. Revista Interuniversitaria de Formación Del Profesorado, 66(23,3), 43-72. Recuperado el día 1 de enero del 2015 de: http://www.aufop.com/aufop/uploaded_files/articulos/1258587233.pdf

Organización Mundial de la Salud (2011) Informe Mundial sobre la discapacidad. Recuperado

de: http://www.who.int/disabilities/world_report/2011/es/

Palacios, A; \& Romañach, J. (2008) El modelo de la diversidad: una nueva visión de la bioética

desde la perspectiva de las personas con diversidad funcional (discapacidad). Revista Sociológica de Pensamiento Crítico. 2(2), 37-47. Recuperado el 06 agosto del 2014 de: http://www.intersticios.es

Rivas, C., Romero, A., Pérez-Llantada, M. d. C., López de la Llave, A., Pourtau, M., Molina, I., González, J. \& Garcia-Mas, A. (2012). Bienestar psicológico, salud general, autonomía percibida y lesiones en futbolistas. Revista de Psicología del Deporte, 21(2) 365-371. Recuperado de http://www.redalyc.org/articulo.oa?id=235126897017

Ryff, C. (1989) Happiness Is Everything, or Is It? Explorations on the Meaning of Psychological WellBeing. Journal of Personality and Social Psychology, 57(6), 1069-1081.

Sandoval, C. (1996). Investigación cualitativa. Bogotá Colombia. ISBN: 958-9329-18-7. Recuperado de http://www.ugr.es/ erivera/PaginaDocencia/Posgrado/Documentos/DisenoInvestigacion.pdf Waterman, A. (1993) Two Conceptions of Happiness: Contrasts of Personal Expressiveness (Eudaimonia) and Hedonic Enjoyment. Journal of Personality and Social Psychology, 64(4), 678-691. DOI: 10.1037/0022-3514.64.4.678 